University of Nebraska - Lincoln

DigitalCommons@University of Nebraska - Lincoln

CSE Conference and Workshop Papers

Computer Science and Engineering, Department

2002

\title{
Dynamic Routing in Translucent WDM Optical Networks
}

\author{
Xi Yang \\ University of Southern California, xyang@isi.edu \\ Byrav Ramamurthy \\ University of Nebraska-Lincoln, bramamurthy2@unl.edu
}

Follow this and additional works at: https://digitalcommons.unl.edu/cseconfwork

Part of the Computer Sciences Commons

Yang, Xi and Ramamurthy, Byrav, "Dynamic Routing in Translucent WDM Optical Networks" (2002). CSE Conference and Workshop Papers. 117.

https://digitalcommons.unl.edu/cseconfwork/117

This Article is brought to you for free and open access by the Computer Science and Engineering, Department of at DigitalCommons@University of Nebraska - Lincoln. It has been accepted for inclusion in CSE Conference and Workshop Papers by an authorized administrator of DigitalCommons@University of Nebraska - Lincoln. 


\title{
Dynamic Routing in Translucent WDM Optical Networks*
}

\author{
Xi Yang and Byrav Ramamurthy \\ Department of Computer Science and Engineering, University of Nebraska-Lincoln \\ 115 Ferguson Hall, UNL, Lincoln, NE 68588-0115
}

\begin{abstract}
Translucent WDM optical networks use sparse placement of regenerators to overcome the impairments and wavelength contention introduced by fully transparent networks, and achieve a performance close to fully opaque networks with much less cost. Our previous study proved the feasibility of translucent networks using sparse regeneration technique. We addressed the placement of regenerators based on static schemes allowing only fixed number of regenerators at fixed locations. This paper furthers the study by proposing a suite of dynamical routing schemes. Dynamic allocation, advertisement and discovery of regeneration resources are proposed to support sharing transmitters and receivers between regeneration and access functions. This study follows the current trend in optical networking industry by utilizing extension of IP control protocols. Dynamic routing algorithms, aware of current regeneration resources and link states, are designed to smartly route the connection requests under quality constraints. A hierarchical network model, supported by the MPLS-based control plane, is also proposed to provide scalability. Experiments show that network performance is improved without placement of extra regenerators.
\end{abstract}

Keywords- Dynamic Routing, Routing and Wavelength Assignment, Regeneration, Optical Network.

\section{INTRODUCTION}

WDM optical networks are providing huge bandwidth to keep up with the explosive growth of the Internet. Although most people are optimistic about the emergence of an alloptical global-scale network, they must address the technical difficulty in overcoming the signal impairments introduced by long-haul fibers and optical components such as Erbium Doped Fiber Amplifiers (EDFAs) and Optical CrossConnects (OXCs). In the near future, regeneration will be a useful method to extend the reach of optical networks. Optical-Electronic (O/E) and Electronic-Optical (E/O) conversion may be indispensable for the control of optical networks. Our research focuses on the efficient utilization of regeneration resources in optical networks [1]. This study

* This work was supported in part by NSF grants (ANI0074121 and EPS-0091900). will introduce dynamic routing schemes into our network architecture and merge it with the trend of using IP control in optical networks. We will show how regeneration resources can contribute to a unified control plane.

\subsection{Previous Work on Translucent Networks}

Transparent optical networks are difficult to be deployed on a large scale. The reason for this is the effect of transmission impairments on the signal quality after a call travels through several optical links and components. There are still no satisfactory methods to overcome the impairments in optical domain. A long-distance lightpath may require electronic signal regeneration, in order to clean it up and improve its quality. An extreme case of this approach is the opaque optical network. An opaque optical network incorporates such signal regeneration at every intermediate node along the lightpath. Considering the huge capacity of multiple wavelengths in a fiber, the cost of an opaque network may be prohibitive. Our previous study suggested a feasible translucent network architecture using sparse regeneration $[2$, 3 , 4]. In a translucent optical network, a signal is made to traverse as long as possible before its quality falls below a threshold value. Because a signal is regenerated only if necessary, we need much less regeneration resources. Experiments showed that about only $20 \%$ of regeneration nodes are needed to achieve a performance close to an opaque network [4].

Figure 1.1 is the regeneration node model in a translucent network. A node consists of an OXC and an access station. Regeneration resources can be represented with the transmitters $\left(\mathbf{T}_{\mathbf{X}}\right)$ and receivers $\left(\mathbf{R}_{\mathbf{X}}\right)$ in the access station. $\mathbf{T}_{\mathbf{X}}$ and $\mathbf{R}_{\mathbf{X}}$ can be statically or dynamically assigned into three sets, $\mathbf{T}_{\mathbf{A}}, \mathbf{R}_{\mathbf{A}}$ and $\mathbf{T}_{\mathbf{R}} \mathbf{R}_{\mathbf{R}}$. If a call is at its source, it enters the $\mathbf{T}_{\mathbf{A}}$ set. If the call is at its destination, it enters the $\mathbf{R}_{\mathbf{A}}$ set. If the call is at an intermediate node, a transmitter-receiver (T-R) pair is in the $\mathbf{T}_{\mathbf{R}} \mathbf{R}_{\mathbf{R}}$ set. The previous study considered a fixed number of $\mathbf{T}-\mathbf{R}$ pairs allocated and reserved for regeneration. Each regenerator is fixed at a node in a network based on regenerator placement algorithms. The placement algorithms must be executed offline, i.e. at the network planning stage. We used Shortest Hop First (SHF) algorithm to calculate routes. Although the sparsely placed regenerators contribute to improving the performance, the routing and wavelength assignment is unaware of the regenerators and hence cannot efficiently utilize them. 


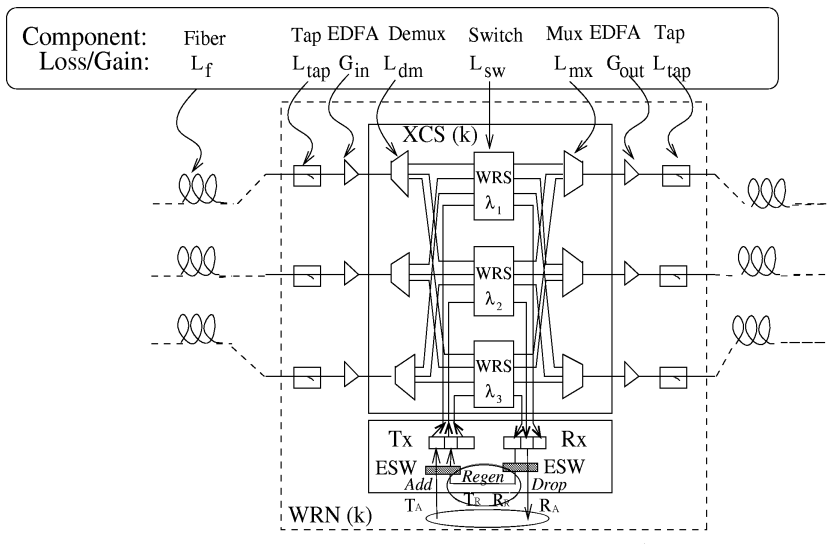

Figure 1.1 Regeneration node model.

We also addressed another demand for regeneration in earlier work [3, 4]. Wavelength contention is one of the primary reasons for blocking calls. A technique for resolution is wavelength conversion. Because wavelength converters implemented completely in optical domain remain expensive and difficult, it is simpler to use the electronic regeneration resources to convert wavelengths. Nodes using the regeneration model in Figure 1.1 can satisfy at least part of such demands. However, the offline allocation of regeneration resources cannot fully utilize their potential.

\subsection{Review of Relevant Study}

Other undergoing research also supports the idea of sparse regeneration in a large-scale optical network. As surveyed by [6], the ordinarily used $600 \mathrm{Km}$ span distance is far from the requirement by today's Internet traffic. Even a reach distance of $3000 \mathrm{Km}$ can only satisfy $60 \%$ of the connections. However, current technology has difficulty to extend the transparent reach distance to more than $2000 \mathrm{Km}$ [5]. In [7], the authors suggested using "islands of transparency" to establish sub-connections between regeneration sites. Similar to our previous study, sub-connections are designed offline based on physical constraints. In [8], the challenge for optical performance monitoring in optical networks was discussed. Our previous regeneration node model (see Figure 1.1) used tapping of the optical channels to monitor the signal quality.

Wavelength routing is often divided into two stages, i.e. routing and wavelength assignment. When talking about routing in this paper, we mean both stages, with an emphasis on the first. The routing issues in the Internet have been studied a lot. Several protocols such as Open Shortest Path First (OSPF), Intermediate System-to-Intermediate System (IS-IS) and Border Gateway Protocol (BGP) are widely used. The basic idea is to make each node maintain its local state by local monitoring. Every node can advertise the local state and exchange information with other nodes to obtain the global state of the network. The Shortest Path First (SPF) algorithm is generally used in route calculation. To meet the requirement of Quality of Service (QoS), some performance metric, such as loss, delay and jitter, can be incorporated with the modified Dijkstra's or Bellman-Ford algorithm to achieve an optimal solution. Recent research efforts have extended these algorithms to route wavelengths in an optical networks [9]. Subject to bandwidth or quality constraints on links, the routing objective can be either a constrained or a minimum metric [10].

Many proposed algorithms use static or offline routing, which calculates routes prior to the existence of connection requests. Instead, dynamic or online routing satisfies the requests one-by-one based on current network states [10,11]. Dynamic routing algorithms are more suitable in an environment where network resources dynamically change and require optimal utilization.

Over the last few years, there has been a trend to develop a unified control plane covering both IP and optical networks under the umbrella of Multiprotocol Label Switching (MPLS) $[12,13]$. Using MPLS signaling (Resource Reservation Protocol (RSVP) and Label Distribution Protocol (LDP)), OXCs can inter-operate with IP Label-Switched Routers (LSRs) to establish a lightpath as a Label Switched Path (LSP) [14]. An additional benefit of MPLS is the hierarchical routing and switching architecture. MPLS supports forwarding hierarchy via label stacking and hence an LSP can be considered as a link in the IS-IS or OSPF link state database. Lightpaths or optical trails can be treated as links to achieve an excellent scalability [13].

\section{TRANSLUCENT DYNAMIC ROUTING}

Regenerators can share resources with access stations. Since traffic cannot use all the transmitters and receivers in access stations, some nodes must have spare T-R pairs for regeneration. In this study, we do not need to place extra number of T-R pairs at fixed nodes. Every node with spare T$\mathrm{R}$ pairs can become a potential regenerator. However, the availability of T-R pairs may change from time to time due to sharing. To cope with the variability, the resources need to be dynamically managed. The available status of regeneration resources needs to be dynamically updated into the global network states. It is impossible for a static or offline algorithm to select efficient routes in this case. Hence dynamic routing algorithms based on the updated network states become a must.

\subsection{Features of Dynamic Routing Algorithms in Translucent}

\section{Networks}

1. Dynamic resources allocation: To share the resources between access and regeneration functions, the resource management module must support a series of operations that can allocate transmitters and receivers into the three sets $\left(\mathbf{T}_{\mathbf{A}}, \mathbf{R}_{\mathbf{A}}\right.$ and $\left.\mathbf{T}_{\mathbf{R}} \mathbf{R}_{\mathbf{R}}\right)$, maintain their status and map them to OXC channels.

2. Link state probe and management: There are two regeneration-specific parameters needed as link states, 
viz., electronic speed rate and format. Furthermore, for QoS and traffic engineering purposes, signal quality metrics, such as Bit Error Rate (BER), will be used in routing subject to a constrained or minimum objective. Collecting link states is a key task for route calculation. We used taps in previous study to collect the signal quality parameters in optical domain. It is complex and costly. The regeneration process allows us to implement the probe in electronic domain. Only minor modification, with low cost, is needed on hardware. Relevant parameters and measurements can be incorporated into the Link Management Protocol (LMP) currently under development [15].

3. Regeneration resource advertisement and discovery: Link-state routing protocols, such as OSPF, have formatted messages to communicate between routers to exchange the sates of adjacent links. In optical networks, with the support of LMP, we need a little modification to the messages to advertise regeneration-relevant information, including bit rate, format, load status, BER, etc.

4. Efficient routing algorithms: When taking the regeneration-specific parameters and quality metric into account, we can modify the Constrained SPF algorithm to satisfy the new objectives. The design of algorithms will be discussed in the later sections.

5. Integration with MPLS-based control plane: The IP community has extended the IP control plane to connection-oriented technology. The work done on MPLS removes the need for invention of a new control plane for optical networks. In this paper, dynamic resource allocation, discovery, link management and constrained routing are designed close to IP control protocols extended under the MPLS umbrella. This guarantees minimal modification in commercial implementations to meet the emerging standards. MPLS can also provide detailed signaling protocol (RSVP/LDP) and advanced options, such as traffic engineering, for prototype design.

6. Hierarchical routing architecture: Generalized MPLS uses label stacking to construct a hierarchical network. Due to the difficulty in stacking labels in optical domain, it was proposed to bind electronic LSPs with optical trails in port mapping tables. A regeneration node can be the control point that bridges the electronic and optical layers. We will have a clear picture of this idea in the next section.

\section{HIERARCHICAL NETWORK MODEL}

In this section we introduce the regeneration layer into the hierarchy of MPLS control plane. MPLS LDP supports this layer by inserting a regeneration-specific label into the label stack. There are several benefits in doing this. First, the inherent scalability of LSP-based hierarchy can make the control plane work for a very large and complex-topology network. Second, when we need specific control on regeneration resources, an independent regeneration layer in the hierarchy can provide necessary separation from other layers by LSP tunneling. Finally, the regeneration layer is tightly coupled to the optical layer and hence at least part of the control functions on optical layer can be shifted to regeneration layer. It is easier to adjust label distribution and stacking in electronic domain. In this study, the control of regeneration layer will be shown to contribute to more efficient routing in a translucent WDM optical network.

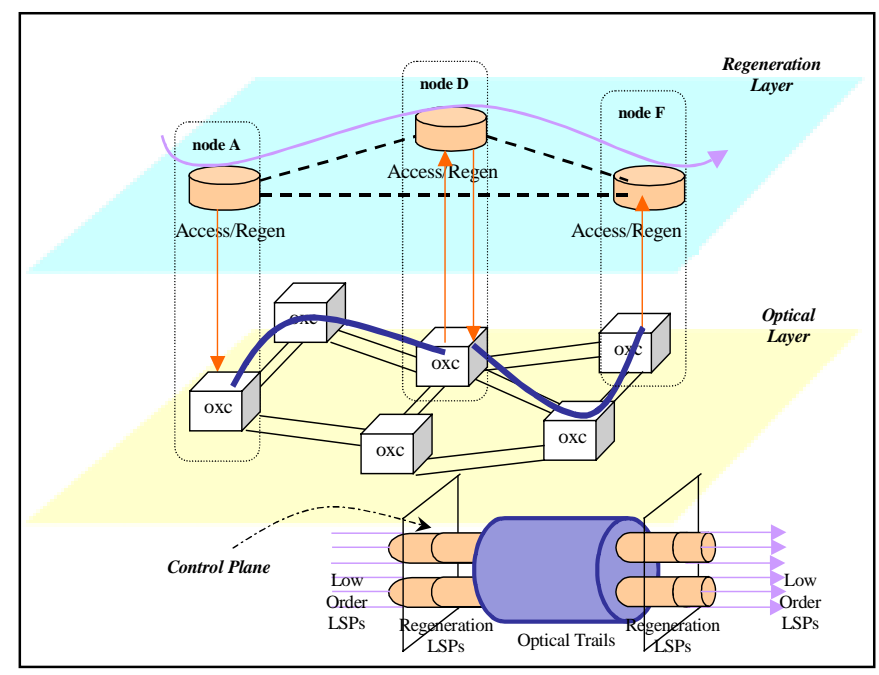

Figure 3.1 Hierarchical translucent network model.

Figure 3.1 plots the hierarchical network model incorporating the regeneration layer. Low-order LSPs can be any electronic path carrying IP, ATM, Frame Relay or SONET traffic. Regeneration LSPs are considered as basic electronic links along the path. A regeneration link is bound to an optical trail or sub-connection, which is the highest LSP in this hierarchy. The optical trails provide capacity for transmission. When a single optical trail cannot satisfy the connection request, a low-order path is routed through the regeneration layer by discovering available regeneration resources. The control plane is extended to where the regeneration resources exist. The access/regeneration module becomes a special signaling control point using MPLS signaling.

Similar to 'regeneration section' in SONET/SDH, regeneration layer is the closest to optical layer in the hierarchy. However, regeneration layer in a translucent WDM optical network has much more responsibility for control. The regeneration resources are managed and dynamically allocated at this layer. As the terminating points of basic electronic links, i.e. regeneration LSPs, access/regeneration modules probe and maintain important quality parameters and other link states. The control plane 
supports the regeneration resource discovery and link state exchanges. The establishment of wavelength routes also needs the presence of regeneration layer using MPLS signaling.

This model provides a platform to execute dynamic routing algorithms in a translucent network. On the one hand, it provides the mechanism to collect regeneration-specific information by dedicating control functions of the regeneration layer. The LSPs connecting regeneration resources at different nodes can be simply treated as links. Hence the routing algorithms process the regeneration LSPs in the same way as the links between traditional electronic routers. On the other hand, with the hierarchical model, the control plane extends MPLS signaling to the regeneration layer, so the calculated routes can be established quickly. The hierarchy model allows controlling the regeneration layer independent of other layers. This prevents the dynamic resource allocation and routing at this layer from interfering with other layers, and provides flexible scalability.

\section{DESIGN OF DYNAMIC ROUTING AlgORITHMS}

The hierarchical translucent network model implies a hierarchical routing architecture. Routing on regeneration layer can be separated from routing on optical layer. MPLS signaling guarantees a unified control and establishes routes through both layers. When a connection can be satisfied without regeneration, it needs only one physical link, which is an optical trail with a continuous wavelength. Otherwise, multiple links or sub-connections are needed using regeneration.

We present the basic ideas of this routing scheme as follows.

1. Optical-layer control discovers available optical trails between access/regeneration modules. Shortest Hop First (SHF) is used to select a continuous wavelength, based OXC connection information supplied by control plane.

2. Access/regeneration module maintains a connection table that stores connectivity information to other access/regeneration modules.

3. The links between access/regeneration modules are dynamically bound to optical trails. A link state table is maintained to store the updated link sates, including supported formats, bit-rate, BER and powers.

4. A connection request triggers the routing algorithm at the source node. The source node uses the latest global link states to calculate the route on the regeneration layer. Constrained Shortest Path First (CSPF) link-state routing algorithm is executed.

We propose two regeneration-layer routing algorithms in this study. Both algorithms incorporate regeneration link states with SPF. One has a minimum number of regeneration hops with a threshold BER constraint. The other has a minimum BER objective with limited regeneration hops. In a hierarchical routing architecture, the regeneration layer has a particular view on the network. Each regeneration link corresponds to a LSP (optical trail). There have been many algorithms addressing all-optical RWA. We assume a Shortest Hop Path (SHP) routing and First-Fit wavelength assignment on the optical layer.

To simplify the design, we ignore the constraints on regeneration bit rate, format and other QoS parameters, e.g. delay and jitter, in this study. We can use the sum of BER value on each link to calculate the total BER on a path because each hop cleans the optical signal and retransmits it with a full power after electronic regeneration.

\subsection{Minimum Regeneration Hops with BER Constraint}

\section{(MRHBC) Routing Algorithm}

Using a general method to model a routing algorithm, the network on the regeneration layer can be viewed as a weighted directed graph $\mathrm{G}(\mathrm{V}, \mathrm{E})$. For dynamical routing, the vertex set $\mathrm{V}$ and the edge set $\mathrm{E}$ can be updated with the regeneration resource discovery scheme. Even the edge weight can vary depending on current probing and measurement on links.

When the objective is a minimum number of regeneration hops, each edge weight equals to one. This routing algorithm is reduced to a simple Shortest Hop First (SHF). The algorithm is iterative by removing the highest BER link if the BER constraint cannot be satisfied. The control flow is described below.

Input: Graph $G(V, E)$, BER constraint $c$, source node $s$ and destination node $t$,

BER value on each link

Output: A path $p$ between $s$ and $t$, regeneration link set $L(s, t)$

\section{Algorithm:}

1 Construct $G(V, E)$ based on the connection and link state tables in node database

2 Assign a weight 1 to each edge in $E$.

3 Use Dijkstra's algorithm to find a shortest hop path $p$ from $s$ to $t$

4 If $p$ Not exist Then

5 End Algorithm

\section{End If}

7 Mark the shortest route $\mathrm{p}$ and sum the BER value of marked links.

8 If $\operatorname{sum}<c$ Then

$9 \quad$ Output $p$ and $L(s, t)$

10 Else 
11 Remove the edge with the highest BER value

12 Goto 3

\section{End If}

14 End Algorithm

\subsection{Minimum BER with Regeneration Hop Constraint}

\section{(MBRHC) Routing Algorithm}

This algorithm has an objective on total BER value of a path. Electronic regeneration only produces linear accumulation of BER on regeneration links along a path. Hence SPF routing is allowed based on BER weight. Regeneration is not always good for signal quality because electronic processing may introduce noise, delay and jitter as well. We have a constraint on the regeneration hops for final paths. The algorithm succeeds only if both BER threshold and constrained number of hops are satisfied. Otherwise, a higher BER below the threshold is allowed to limit hops via an iterative procedure.

Input: Graph $G(V, E)$, BER threshold $c$, regeneration hop limit $h$, source node $s$ and destination node $t$, BER value $b(i)$ on each link

Output: A path $p$ between $s$ and $t$, regeneration link set $L(s, t)$

\section{Algorithm:}

1 Construct $G(V, E)$ based on the connection and link state tables in node database

2 Assign weight $b(i)$ to each link/edge $e(i)$ in $\mathrm{E}$

3 Use Dijkstra's algorithm to find a shortest path $p$ from $s$ to $t$

\section{If $p$ Not Exist Then}

5 End Algorithm

\section{End If}

7 Mark the shortest route $p$ and sum the BER value of marked links.

8 If $\operatorname{sum} \geq c$ Then

9 End Algorithm

10 Else If hops of $p>h$

11 Remove the edge/link with the lowest BER value

12 Goto 3

13 Else

14 Output $p$ and $L(s, t)$

15 End If

16 End Algorithm
The output of the routing algorithm is passed to signaling module in the control plane to establish a connection. Each regeneration link in $\mathrm{L}(\mathrm{s}, \mathrm{t})$ will trigger an update on the other layer. Because the bound optical trail is occupied by a subconnection, a new wavelength and probably a new route will be calculated on the optical layer, following which the link rebinding and probably the link removal are executed by the control plane using MPLS. The feedback leads to updating link states on the regeneration layer. Then new connection requests will be routed based on the updated link states. The routing algorithm is dynamically repeated in the translucent networks for all connection requests.

\section{Simulation AND PeRformance ANALYsis}

In this section, we study the performance of dynamic routing algorithms with a proven optical network simulation system, SIMON [16]. We conduct the experiments on two target networks. The first is the Pacific Bell mesh network with 15 nodes and 21 links (see Figure 5.1). The link length varies from $100 \mathrm{~km}$ to $300 \mathrm{Km}$. The second is a 12-node bidirectional ring (see Figure 5.2), whose links have an equal length of $100 \mathrm{~km}$. Each link supports 4 wavelengths. In our previous study, fixed number of regenerators were placed at specific nodes. To form the basis of comparison, we place 3 regenerators at node $0,5,6$ in the Pacific Bell network and 2 regenerators at node 1,7 in the 12-node ring. Each regenerator has 2 transmitter-receiver pairs. Based on the static placement, the SHF routing and First-Fit wavelength assignment algorithms are used. To contrast with the static SHF, the regeneration-layer dynamic routing algorithms incorporated with the optical-layer SHF and First-Fit are implemented in SIMON. Both MBRHC and MRHBC routing algorithms are simulated without any extra regeneration resources ( $T-R$ pairs). The common simulation parameters are listed in Table 5.1.

We define the path length as the number of optical hops/links that a route travels. As has been said, a regeneration hop/link may correspond to multiple optical hops/links. For an average length of 3 optical hops, the Pacific Bell network accommodates a maximum load of (21 $\times 2 \times 4) / 3=56$ Erlangs while the 12-node ring accommodates 32 Erlangs. Figure 5.3 plots the blocking probability of static SHF, MBRHC and MRHBC routing algorithms for the Pacific Bell network under loads ranging from 2 through 50 Erlangs. For the 12-node ring, the loads range from 2 through 30 Erlangs (Figure 5.4).

Figure 5.3 and 5.4 indicate that both MBRHC and MRHBC dynamic routing algorithms have significant improvement on performance when compared with the static SHF routing algorithm. Table 5.2 shows the detailed simulation results under some load for the two target networks. It is obvious that the benefit of regeneration-layer dynamic routing lies in its greatly reducing the blocked calls due to unacceptable BER. This confirms the basic idea of using dynamic resource discovery and link-state aware 
routing to optimize the regeneration resource utilization. The blocking due to wavelength and source/destination contention increases slightly because more accepted calls in turn cause greater contention.

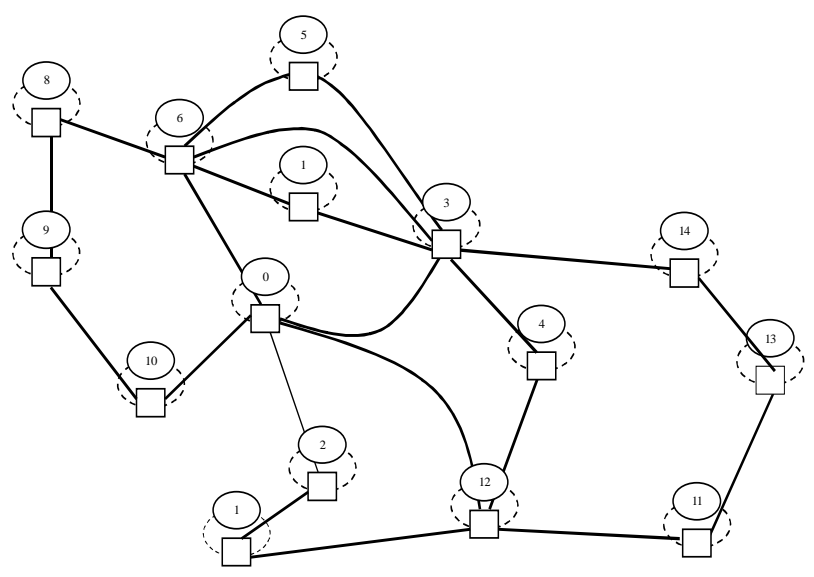

Figure 5.1 Pacific Bell network.

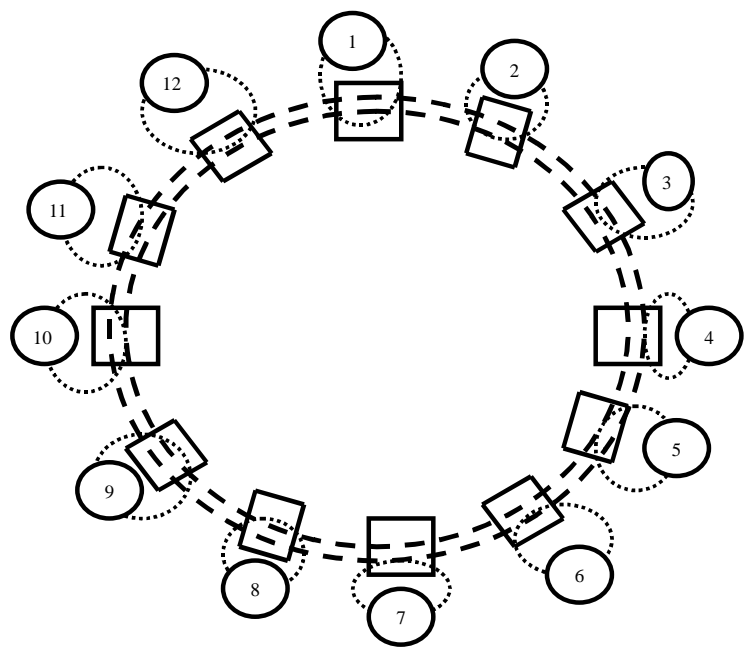

Figure 5.2 12-node bidirectional ring.

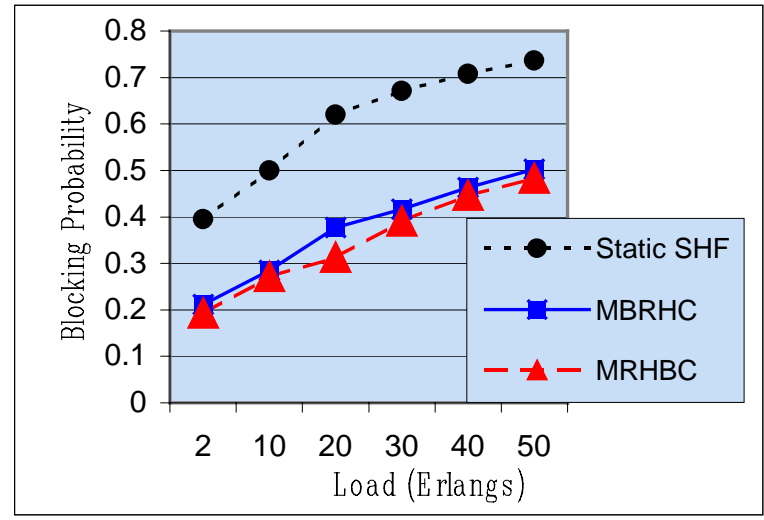

Figure 5.3 Dynamic vs. static routing algorithms on Pacific Bell network.

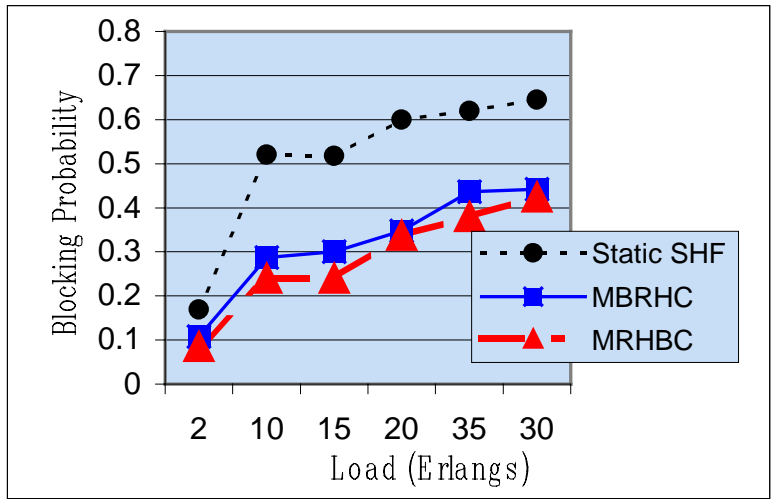

Figure 5.4 Dynamic vs. static routing algorithms on 12-node bidirectional ring.

Table 5.1Common Simulation Parameters.

\begin{tabular}{|c|c|}
\hline Parameter & Value \\
\hline Call Arrivals & Poisson \\
\hline Call Holding Time & Exponential \\
\hline Distribution of Src-Dest Pairs & Uniform \\
\hline Offered Calls & 10,000 \\
\hline BER-threshold Value & $10^{\wedge}-12$ \\
\hline Bit rate per channel (r) & $1 \mathrm{Gbps}$ \\
\hline Wavelength spacing & $100 \mathrm{GHz}$ \\
\hline Mu/Demultiplexer Loss & $4 \mathrm{~dB}$ \\
\hline Input/Output EDFA Gain & $22 \mathrm{~dB} / 16-18 \mathrm{~dB}$ \\
\hline ASE factor & 1.5 \\
\hline Switch Loss & $5 \mathrm{~dB}$ \\
\hline Switch crosstalk Ratio & $-30 \mathrm{~dB}$ \\
\hline Fiber Loss & $0.2 \mathrm{~dB} / \mathrm{km}$ \\
\hline
\end{tabular}

Table 5.2 Detailed simulation results (offered 10,000 Calls).

\begin{tabular}{|c|c|c|c|}
\hline $\begin{array}{l}\text { Pac.Bell (20Erlangs) } \\
\text { / Ring (15Erlangs) }\end{array}$ & Static SHF & MBRHC & MRHBC \\
\hline $\begin{array}{c}\text { Blocked Calls Due to } \\
\text { high BER }\end{array}$ & $6152 / 4531$ & $3682 / 2844$ & $2906 / 2459$ \\
\hline $\begin{array}{c}\text { Blocked Calls Due to } \\
\text { Contention }\end{array}$ & $116 / 673$ & $205 / 331$ & $183 / 247$ \\
\hline 1-hop Offered & $2057 / 1810$ & $1932 / 1535$ & $1905 / 1490$ \\
\hline 1-hop Blocked & $743 / 512$ & $628 / 453$ & $603 / 387$ \\
\hline 2-hop Offered & $3435 / 1762$ & $3136 / 1598$ & $3153 / 1603$ \\
\hline 2-hop Blocked & $1896 / 724$ & $1053 / 497$ & $188 / 432$ \\
\hline 3-hop Offered & $3152 / 1857$ & $2650 / 1802$ & $2327 / 1892$ \\
\hline 3-hop Blocked & $2353 / 873$ & $1136 / 585$ & $765 / 573$ \\
\hline 4-hop Offered & $1249 / 1862$ & $1109 / 1643$ & $1221 / 1656$ \\
\hline 4-hop Blocked & $1142 / 1090$ & $529 / 764$ & $353 / 530$ \\
\hline ( $\mathbf{2 5}$ hops) Offered & $107 / 2709$ & $1073 / 3422$ & $1294 / 3359$ \\
\hline ( $\mathbf{5}$ hops) Blocked & $107 / 2005$ & $541 / 876$ & $484 / 784$ \\
\hline
\end{tabular}


Another observation is that the accepted calls tend to be routed via longer path lengths. The number of calls using more than 3 optical hops in MBRHC and MRHBC is greater than that of the static SHF. Furthermore, for the long-path calls, the regeneration-layer dynamic routing algorithms result in much less blocking probability than the static SHF routing algorithm. The reason is that the dynamic routing algorithms in a hierarchical network can calculate more optimal, and hence possibly more complex, routes than the static routing algorithm in a flat network.

\section{CONCLUSIONS}

This paper contributes a novel idea that incorporates dynamic routing and MPLS-based control plane with sparse regeneration in WDM optical networks. We present our main conclusions below.

1. It is possible to dynamically share electronic resources between regeneration and access functions in a translucent WDM optical network. Using IP-like link state advertisement and discovery schemes, the global states of the network can be updated based on available regeneration resources and link states.

2. It is both desirable and feasible to design the control plane of a translucent WDM optical network under the umbrella of generalized MPLS. We propose an MPLSbased hierarchical network model that supports both the regeneration and optical layers with LSPs using label stacking, which provides flexible scalability.

3. Based on MPLS hierarchy, we propose the dynamic routing algorithms on the regeneration layer independent on the optical layer. Being aware of regeneration resources and link states, the dynamic routing algorithms can select routes smartly, subject to constrained and/or minimum objectives.

4. Simulation shows that the overall blocking probability using dynamic routing algorithms decreases drastically when compared to the static SHF routing algorithm under different network loads in both mesh and ring networks. In particular the blocked calls due to unacceptable BER are greatly reduced. Supported by the regeneration layer, longer paths are more frequently selected with lower blocking probability. It is especially noticeable that the improvement is achieved without placing any extra transmitter-receiver (T-R) pairs, i.e., the actually needed $T-R$ pairs are reduced.

This study furthers the concept of translucent WDM optical networks in our previous study, which addressed the signal impairments and wavelength contention via sparse regeneration in a large-scale WDM optical network. With dynamic routing algorithms and MPLS-based control plane, a translucent WDM optical network using sparse regeneration is closer to commercial use. The dynamic routing algorithms proposed in this study are not aimed to be fully optimized and cannot guarantee complete efficiency. Nevertheless they do prove the potential value of dynamic routing in a translucent WDM optical network. For future study, we propose to combine more factors, such as regeneration bit rate, format and other QoS parameters, in routing constraints and objectives and propose detailed control information into MPLS extensions.

\section{REFERENCES}

1. B. Ramamurthy, H. Feng, D. Datta, J. P. Heritage, and B. Mukerjee, "Transparent vs. opaque vs. translucent wavelength-routed optical networks", Optical Fiber Communication (OFC '99) Technical Digest, San Diego, CA, Feb. 1999.

2. S. Yaragorla, "Sparse regeneration on a translucent WDM optical network", Master Thesis, University of Nebraska-Lincoln, Lincoln, May 2000.

3. B. Ramamurthy, S. Yaragorla, X. Yang, "Translucent Optical WDM Networks for the Next-Generation Backbone Networks", in Proceedings, IEEE Globecom 2001, San Antonio, TX, Nov. 2001.

4. X. Yang and B. Ramamurthy, "Sparse Regeneration in a Translucent WDM Optical Network", Asia-Pacific Optical and Wireless Communications, Beijing China, Nov. 2001.

5. R. S. Vodhanel, et al., Post Deadline Paper PD27, OFC '97, Dallas, TX, Feb. 1997.

6. R. E. Wagner et al., "The Potential of Optical Layer Networks", OFC 2001, Anaheim, CA, March 2001.

7. A. A. Saleh et al., "Proposed Extensions to the UNI for Interfacing to a Configurable All-Optical Network", OIF 2000.278.

8. R. E. Wagner, LEOS Topical Meeting on Broadband Optical Networks Paper FC1.1, Aventura, FL, Jul. 1998.

9. H. Zang, J. P. Jue, and B. Mukherjee, "A review of routing and wavelength assignment approaches for wavelength-routed optical WDM networks", SPIE/Baltzer Optical Network Magazine, vol. 1, no. 1, pp. 47-60, Jan, 2000.

10. S. Chen and K.Nahrstedt, "An overview of Quality-of-Service Routing for the Next Generation High-Speed Networks: Problems and Solutions", IEEE Network, Special Issue on Transmission and Distribution of Digital Video, Nov./Dec. 1998.

11. M. Kodialam and T. V. Lakshman, "Integrated Dynamic IP and Wavelength Routing in IP over WDM Networks", Infocom 2001.

12. E. Rosen, A. Viswanathan, and R., Callon, "Multiprotocol Label Switching Architecture," draft-ietf-mpls-arch-07.txt, July 2000, work in progress.

13. A. Banerjee et al, "Generalized Multiprotocol Label Switching: An Overview of Rouging and Management Enhancement", IEEE Communications Magazine, Jan. 2001.

14. D. O. Awduche et al, "Multi-Protocol Lambda Switching: Combining MPLS Traffic Engineering Control with Optical Crossconnects", Internet Draft draft-ietf-awduche-mpls-te-optical00.txt, Oct. 1999.

15. J. P. Lang, et al., "Link Management Protocol," Internet draft, draftietf-mpls-lmp-00.txt, Aug. 2000, work in progress.

16. B. Ramamurthy, D. Datta, H. Feng, J.P. Heritage, and B. Mukherjee, "SIMON: A Simulator for Optical Networks", the Fifth annual Conf. on All-Optical Networking (part of the SPIE Photonics East'99, Sept. 1999 (invited paper). 